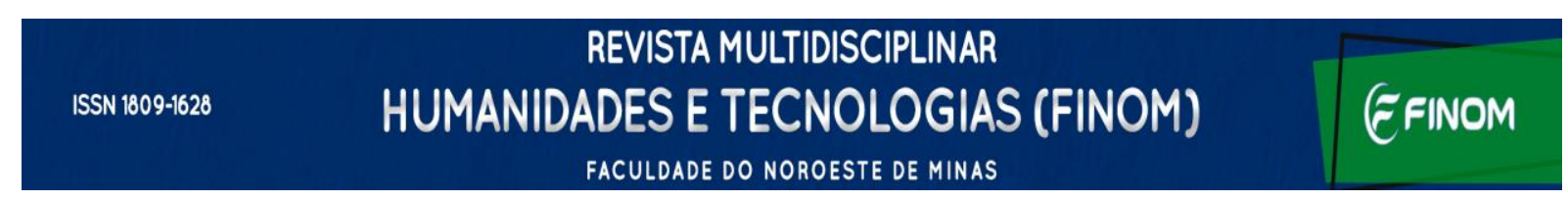

\title{
Gamificação no Ensino Superior em saúde
}

\section{Gamification in health higher education}

\author{
Nielce Meneguelo Lobo da Costa ${ }^{1}$ \\ Carlos Alexandre Felício Brito ${ }^{2}$ \\ Susana Nogueira Diniz ${ }^{3}$
}

\begin{abstract}
Resumo: Este artigo refere-se a uma pesquisa cujo objetivo foi identificar a disseminação do conhecimento sobre gamificação como inovação pedagógica no Ensino Superior em Saúde. A metodologia foi revisão bibliométrica, baseada em Araújo e em Silva, Hayashi e Hayashi. O procedimento metodológico foi o de identificar pesquisas internacionais na base PubMed®, pela incidência de palavras-chave nos textos; dispersão das publicações nos periódicos e frequência de autores e co-autores. As análises foram com o software VOSViewer. Foram identificados 51 periódicos publicados no tema, com maior número de artigos no International Journal of Environmental Research and Public Health. Os artigos envolveram 335 autores, 8 deles com pelo menos duas publicações. Em conclusão, a despeito da importância do tema, ainda não se identifica a existência de uma rede colaborativa em inovação pedagógica no Ensino Superior, na área da Saúde.
\end{abstract}

Palavras-chave: Inovação Pedagógica. Jogos. Bibliometria. Ensino em Saúde

\begin{abstract}
This research aims to identify the knowledge dissemination about gamification as a pedagogical innovation in Higher Education, in Health. The methodology was a bibliometric review, based on Araújo and Silva, Hayashi and Hayashi. The methodological procedure was to identify international searches in the PubMed® database, based on the incidence of keywords in the texts, dispersion of publications in journals and frequency of authors and coauthors. Analyzes were performed with VOSViewer software. 51 journals published on the topic were identified, with the highest number of articles in the International Journal of Environmental Research and Public Health. The articles involved 335 authors, 8 of them with at least two publications. In conclusion, despite the importance of the theme, the existence of a collaborative network in pedagogical innovation in Higher Education in Health has not yet

\footnotetext{
1 Mestrado Acadêmico em Ensino de Ciências e Saúde, Universidade Anhanguera de São Paulo.E-mail nielce.lobo@gmail.com.E-mail: susana.diniz@anhanguera.com

${ }^{2}$ Mestrado Profissional em Inovação no Ensino Superior em Saúde (PPGES), Universidade Municipal de São Caetano do Sul. E-mail: carlos.brito@online.uscs.edu.br

${ }^{3}$ Mestrado Acadêmico em Ensino de Ciências e Saúde, Universidade Anhanguera de São Paulo.
}

Recebido em 23/01/2022

Aprovado em 10/02/2022

Sistema de Avaliação: Double Blind Review

OPEN ACCESS 
政

\section{HUMANIDADES E TECNOLOGIAS (FINOM)}

FACULDADE DO NOROESTE DE MINAS

been identified.

Keywords: Pedagogical Innovation. Games. Bibliometrics. Teaching in Health

\section{Introdução}

Inovar é transformar, introduzir mudanças capazes de efetivar novos procedimentos, disponibilizar diferentes serviços e enfrentar desafios de atualização e competitividade (DRUCKER, 2000). Inovações são promotoras de impactos no desenvolvimento e na viabilidade econômica das Organizações, tais impactos podem ser tanto positivos quanto negativos para o desenvolvimento das Organizações.

Inovar, em qualquer Organização, depende da iniciativa e comprometimento das pessoas, dos indivíduos, das equipes, dos times que as integram. São as pessoas que colocam em ação ideias transformando-as em produtos e/ou resultados. Entretanto, "para inovar é necessária uma combinação de sinergia e inteligência, mas também é preciso condições de estrutura organizacional e um ambiente de acolhimento, confiança, suporte e rapidez de decisões" (LOBO DA COSTA, RAMOS, 2020, p.5).

Nas instituições escolares, inovações pedagógicas são as ações que transformam os processos de ensino e de aprendizagem. Elas alteram a estrutura, modificando métodos e procedimentos, os papeis dos atores escolares e, enfim, sua dinâmica de funcionamento.

No Ensino Superior, um dos desafios para que as inovações pedagógicas sejam implementadas é o despreparo do professor referente às novas metodologias de ensino, aos recursos tecnológicos educacionais e às maneiras de promoção das avaliações.

A sociedade atual, caracterizada pela fluidez, incertezas e fugacidade, como ensina Baulman (2013), apresenta demandas educacionais que devem ser consideradas no processo de ensino, esse é um outro, que traz consigo diversas questões, desde as culturais e as tecnológicas, até a forma como se desenvolve o trabalho docente.

Assim, percebe-se a necessidade e urgência de formação pedagógica para professores universitários que atuam em cursos das mais variadas áreas e, em particular, na área da Saúde. Formação para auxiliá-los, no Ensino Superior a subsidiar o preparo dos futuros profissionais atuantes na Saúde, de modo a atender as demandas da socidade atual.

Focando na premência de se discutir, nos processos formativos, metodologias de 


\section{REVISTA MULTIDISCIPLINAR

ensino inovadoras para o Ensino superior, identifica-se os jogos digitais e a gamificação como possibilidades, não apenas com as características de ludicidade e de aprendizado para o Ensino Infantil, Ensino Fundamental e Médio mas, também, para o Ensino Superior.

Assim sendo, neste artigo, o objetivo foi identificar a disseminação do conhecimento sobre jogos digitais e gamificação como inovação pedagógica no Ensino Superior, na área da Saúde. Para tanto, apresentamos uma revisão bibliométrica da literatura referente a estudos internacionais sobre o tema.

\section{Fundamentação teórica}

A fundamentação teórica da pesquisa é formada pelo conceito de jogo, da sua utilização na área educacional e da gamificação como uma metodologia para aprendizagem no Ensino Superior, particularmente na formação de futuros profissionais para atuação na área da Saúde.

O jogo e a gamificação

O Ensino Superior no Brasil, em especial nos cursos de Ciências da Saúde, vem passando por transformações substanciais nas últimas décadas. Muitas delas estão relacionadas à mudança da estrutura curricular para modelos menos compartimentados e implantação de metodologias de ensino, nas quais o aluno é colocado em posição de protagonismo no processo de aprendizagem. Dentre os métodos utilizados estão modelos, tais como: o aprendizado baseado em problemas (PBL), aprendizado baseado em equipes (TBL) e simulações realísticas (AZEVEDO; PACHECO; SANTOS, 2019). Entre as propostas estudadas e aplicadas está a gamificação (LANDERS, 2014; ORTIZ-COLÓN; JORDÁN; AGREDAI, 2018), que visa a utilização de elementos de jogos para fins educacionais.

Apesar de um crescente interesse em atividades pedagógicas gamificadas, ainda existem muitas lacunas na forma como o conteúdo instrucional e os elementos de jogos podem dialogar, como sistematizar esse diálogo e qual a perspectiva de resultado desse movimento. Assim sendo, são necessários estudos com ênfase no design, aplicação prática e objetivos pretendidos com a atividade gamificada (MAJURI; KOIVISTO; HAMARI, 2018).

"Jogo" é uma palavra polissêmica com significados que mudam de acordo com o 
contexto histórico e a cultura, porém fica claro pelo estudo do fenômeno que todo jogo se presta a um objetivo, ou seja, sempre está imbuído de uma função significante que é atribuída pelos jogadores, como discutido pelo filósofo Huizinga (2000, p. 5) na sua obra "Homo Ludens" de 1938:

O jogo é mais do que um fenômeno fisiológico ou um reflexo psicológico. Ultrapassa os limites da atividade puramente física ou biológica. É uma função significante, isto é, encerra um determinado sentido. Ao jogar, existe para o indivíduo alguma coisa 'em jogo' que transcende as necessidades imediatas da vida e confere um sentido à ação. Todo jogo significa alguma coisa para quem joga.

Huizinga define um jogo como uma atividade realizada voluntariamente em um espaço físico e de tempo definido, seguindo regras obrigatórias e consentidas, visando um fim em si mesma. De forma semelhante Roger Caillois descreve em sua obra "Os jogos e os homens" (1958) o jogo como uma atividade voluntária, com espaço e tempo definidos, com resultado incerto, não gerador de bens, regido por regras convencionadas e em uma realidade "a parte" da vida real (CAILLOIS, 2001).

No século XXI, mas ainda com definição semelhante às apresentadas pelos teóricos anteriores, Deterding et al. (2011), caracterizam jogos por regras e competição (ou esforço) visando um desfecho ou objetivo, sendo executado por participantes humanos. Além disso, os autores concluem que a definição de jogo deve ultrapassar suas propriedades óbvias, e incluir significados construídos situacional e socialmente. Vale ressaltar que, na atualidade, há uma ampliação em relação aos participantes do jogo, que podem envolver não-humanos.

Outros autores, tais como Salen e Zimmerman (2012, p. 95), definem jogo como “[...] um sistema no qual os jogadores se envolvem em um conflito artificial, definido por regras, que implica um resultado quantificável" e separam o jogo propriamente dito dos diversos tipos de interações que ocorrem em razão dele, as quais passam a receber o nome de "interação lúdica significativa" ou "gameplay".

Os atributos dos jogos com fins educacionais e a interação com os jogadores foram inicialmente tipificadas por Wilson et al (2009), os quais identificaram dezoito categorias, posteriormente agrupadas por Bedwell et al (2012), em nove atributos básicos de interação que são potencialmente relevantes para promover aprendizagem. São eles: (1) Linguagem de ação - adaptada, modelada de acordo com a habilidade do jogador e a dificuldade do jogo, (2) avaliação - ao longo do processo de jogar, (3) conflito/desafio - interações diretas ou indiretas 


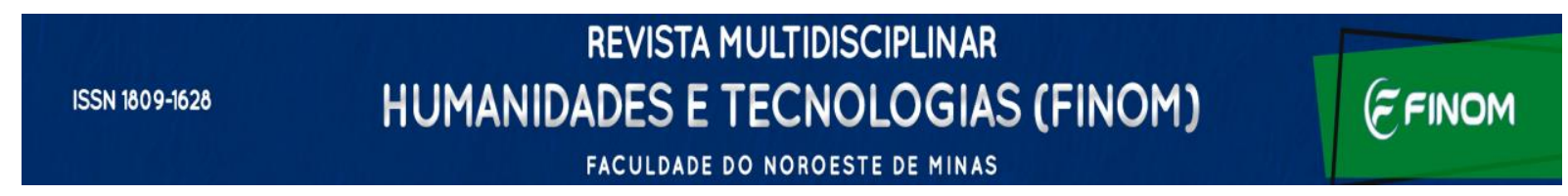

que podem ser conflituosas e desafios para aquele tipo de educando, (4) controle - ligado ao poder, à fantasia ou a influência sobre os elementos do jogo, (5) ambiente - com cenários e personagens inusitados, (6) ficção de jogo - fantasia, mistério, (7) interação humana interação interpessoal, intrapessoal e social (com outros jogadores, com o jogo, ou com o grupo), (8) imersão - estimulos sensoriais, segurança, curiosidade, surpresas, ideias e (9) regras/objetivos - regras de funcionamento do jogo e as metas para vencer, cumprir a tarefa ou desempenhar a ação.

Esses nove atributos devem ser considerados ao se criar ou utilizar jogos por educadores. Nos jogos ditos serious game ou "jogos sérios" o objetivo é aprendizagem e não apenas entretenimento, assim sendo, os fins pedagógicos devem preceder a escolha dos elementos de gamedesign.

Em conclusão, independente do período histórico e enfoque pelo qual o fenômeno é analisado pode-se perceber características constantes, direta ou indiretamente, nas diversas definições, tais como: participação voluntária, tempo e espaço definidos, interação de algum tipo, regras obrigatórias e consentidas, e um resultado final verificável. Jogar envolve aprendizado e o desenvolvimento de estratégias para vencer ou resolver a situação problema apresentada.

\section{Metodologia}

A revisão aqui apresentada utilizou o método bibliométrico, o qual envolve medir, compreender e avaliar as pesquisas identificadas nas buscas, utilizando técnicas quantitativas para mensurar a produção e a disseminação científica. (SILVA, HAYASHI e HAYASHI, 2011)

O método se estrutura a partir de três leis fundamentais, a saber:

1. Lei de Lotka: Refere-se a medir a produtividade de cada autor de artigo nos periódicos da área de investigação. Por meio dessa produtividade se identificam os principais investigadores (autores e co-autores) na temática e no campo da pesquisa relacionada à ela, assim como as redes de colaboração entre pesquisadores.

2. Lei de Bradford: Consiste em medir a dispersão da produção nas diferentes revistas científicas e periódicos na temática em pauta. Parte do pressuposto que as publicações são relevantes para revelar e disseminar os resultados encontrados no campo da pesquisa de um 
tema em uma área do conhecimento. A análise da produção publicada nos periódicos qualificados de uma área pode revelar ao longo do tempo, as tendências da área, quais as comunidades acadêmicas que estão se dedicando à pesquisa na área e como o conhecimento está sendo aprofundado.

3. Lei de Zipf. Consiste em identificar a frequência de ocorrência das palavras em um texto. Vale-se de palavras-chave citadas, resumos e textos completos. Por meio dessas frequências é possível estabelecer conexões e relações nos temas pesquisados em uma área do conhecimento. Novamente, é possível analisar a evolução, ao longo do tempo, das temáticas que são objeto de pesquisa e das formas usadas pelos pesquisadores para disseminação dos resultados encontrados.

Nesta pesquisa, a partir dos fundamentos teóricos e da proposição das leis fundamentais para revisão, foram definidos os indicadores bibliométricos da atividade científica e tecnológica - baseados nas contagens de publicações, citações, co-autores - ou os indicadores relacionais - baseados na contagem de co-autores, co-citações, índice de afinidade. São exemplos de indicadores bibliométricos: fator de impacto, número de citações, mapas dos países e dos campos científicos. (OKUBO, 1997)

Vale ressaltar que, segundo Araújo (2006), o método bibliométrico não permite interpretar precisamente os indicadores por ele produzidos, implicando na necessidade de uso de outros métodos, tais como o sistemático e o integrativo, assim como a associação com os referenciais teóricos. Entretanto, pelas limitações próprias de um artigo, apresenta-se aqui parte da pesquisa, no caso a referente à revisão bibliométrica.

Optou-se por desenvolver a revisão bibliométrica da literatura utilizando o banco de dados PubMed $\AA^{4}$ Publication in Medicine, do National Library of Medicine integrado ao National Center for Biotechnology Information, uma vez que congrega publicações da área da Saúde. O idioma referenciado foi o inglês e a busca foi sem estabelecimento de filtros para restringir os indicadores do banco, quais sejam: país, data de publicação, tipo de artigo, disponibilidade do texto.

O procedimento metodológico foi o de identificar na referida base PubMed® a frequência de autores e co-autores, a dispersão das publicações nos periódicos e a incidência

\footnotetext{
${ }^{4}$ PubMed $\circledast$ comprises more than 32 million citations for biomedical literature from MEDLINE, life science journals, and online books. Citations may include links to full text content from PubMed Central and publisher web sites. PubMed is a free resource supporting the search and retrieval of biomedical and life sciences literature with the aim of improving health-both globally and personally.

https://pubmed.ncbi.nlm.nih.gov/?cm
} 


\section{REVISTA MULTIDISCIPLINAR \\ ISSN 1809-1628 \\ HUMANIDADESE TECNOLOGIAS (FINOM)

de palavras-chave nos textos (designadas pelos autores). Foi considerado para a análise, na dispersão das publicações, uma frequência de publicações nos periódicos de pelo menos duas vezes. Os descritores foram: Ensino Superior, Gamificação e operadores booleanos OR e AND. Assim, a busca foi feita - em 10 de agosto de 2021 - para localizar artigos com a expressão Higher Education OR Undergraduate AND Gamification.

As análises utilizaram o software VOSViewer (https://www.vosviewer.com/), versão 1.6. para o estabelecimento das relações entre os indicadores.

\section{Resultados e discussão}

Foram localizados 73 artigos, vinte e sete deles apresentam no título menção à Education e vinte à Learning, cinco mencionam E-learning, cinco referem-se à Physical Activity, quatro envolvem Motivation, seis abordam Serious Games, três são relativos à Medical Education. Esses artigos envolveram 335 autores, entre esses 8 têm pelo menos dois artigos publicados na temática.

Figura 1 - Redes locais de Autores e co-autores com duas ou mais publicações

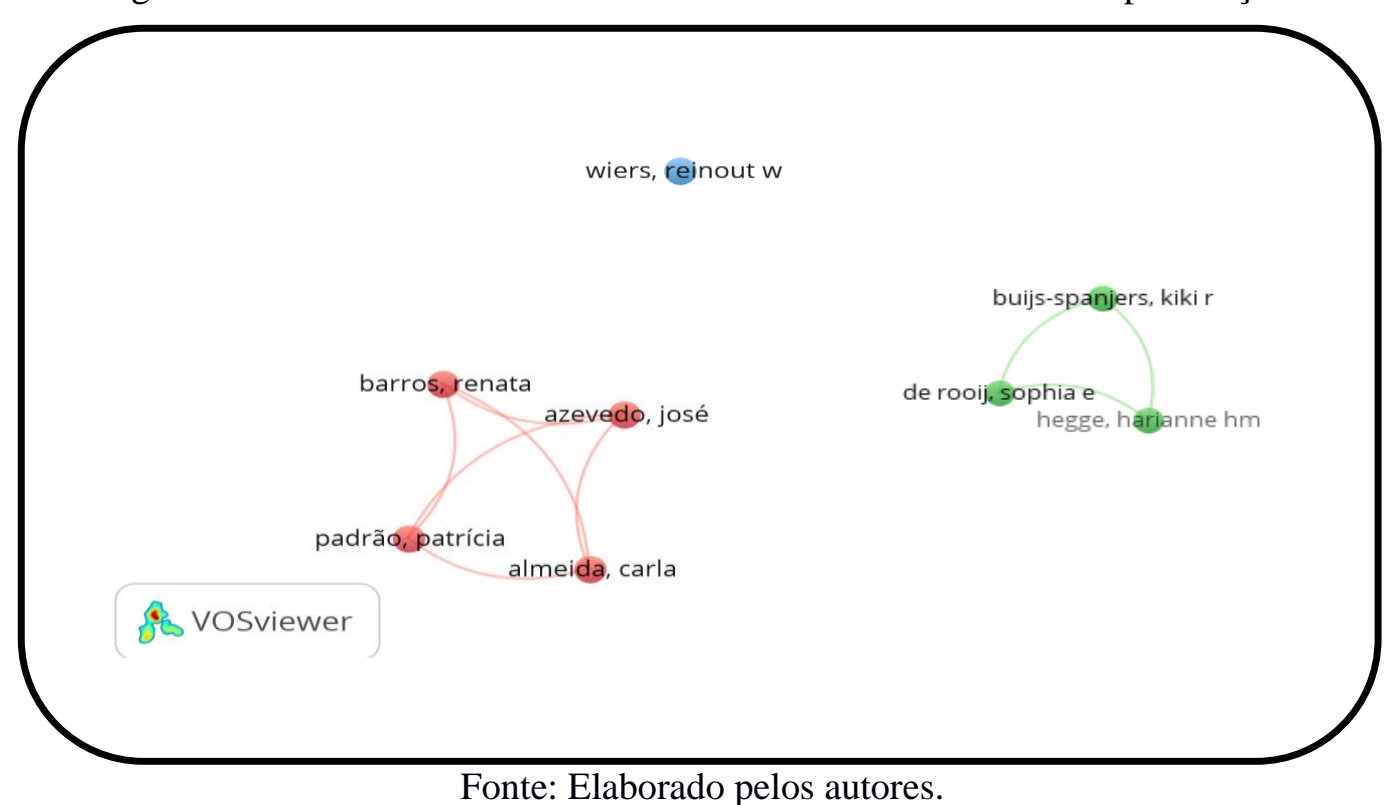

Investigando a relação de co-autoria foram geradas duas redes locais de autoria, sem links entre elas (Figura 1). Um terceiro autor surgiu também com uma incidência de mais de um artigo com outros autores, entretanto sem co-autoria que caracterizasse a formação de uma rede colaborativa. 


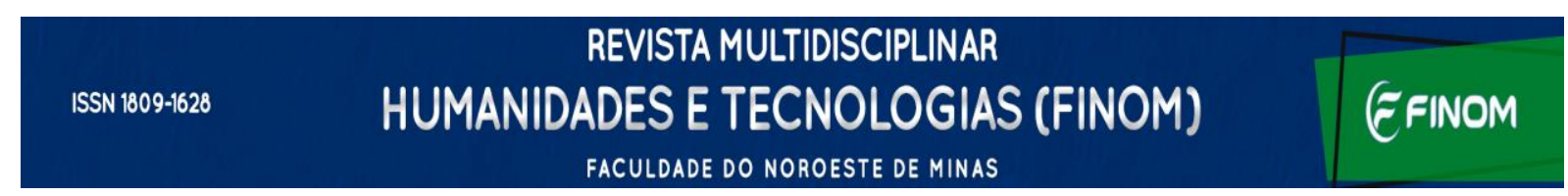

A primeira rede (em vermelho) é constituída por quatro autores portugueses, a saber: Azevedo, J.; Padrão, P., Guegório, Barros, R.; Almeida, C. Apresentando duas publicações em comum, nos anos de 2019 e 2021. Tais artigos são da área da Nutrição, ambos relativos a um Programa denominado "Nutriscience Project". A primeira publicação discute o Programa quanto às possibilidades viabilizadas por ele para impulsionar conhecimentos nutricionais em famílias com filhos de 3 a 5 anos. O artigo é do Journal of Nutrition Education and Behavior: Advancing Research, Practice \& Police, 2019. (AZEVEDO et al, 2019). A publicação seguinte foi relativa a práticas, preferências, habilidades e atitudes dos pais em relação ao consumo alimentar de crianças em idade pré-escolar e foi publicado no Journal PLoS One, 2021. (ALMEIDA C et al, 2021).

Observa-se que a rede só foi estabelecida devido à co-autoria desses quatro autores, em pelo menos duas publicações.

A segunda rede (em verde) é formada por três autores, a saber: Buijs-spanjers, K.; De Rooij, S.; Hegge, H. Apresentando duas publicações em comum, nos anos de 2018 e 2020. Tais artigos são da área da Medicina, relativas a intervenções educacionais para estudantes de Medicina com o uso de um jogo sério. No primeiro os autores relatam a análise de jogos para abordar experiência de Delirium em pacientes, estabelecendo a adequação de uso de um jogo convencional e um jogo sério (Delirium Experience) para estudantes ampliarem conhecimentos sobre geriatria. A publicação está em revista especializada em jogos sérios, qual seja: JMIR Serious Games (BUIJS-SPANJERS, K.R. et al, 2018). A publicação seguinte foi relativa a experiências de aprendizagem, a partir de diferentes estratégias de estudantes de medicina enquanto jogavam o jogo sério Delirium Experience. O artigo foi também publicado na revista JMIR Serious Games (BUIJS-SPANJERS, K.R. et al, 2020).

Observa-se que esta rede só foi estabelecida devido à co-autoria desses três autores, em pelo menos duas publicações.

$\mathrm{O}$ autor Wiers, R. aparece isolado na figura 1, em azul, com duas publicações nos anos de 2015 e 2016, também na temática dos jogos sérios. A primeira publicação é sobre uma pesquisa com jogo sério, que objetiva evitar comportamentos de risco para consumo de álcool. O jogo, intitulado Approach Avoid Task (AAT), tem como fundamento a autoeficácia e foi aplicado para alunos de graduação com tendência a o comportamento de risco. O jogo envolve cenários e alguns deles devem ser rapidamente evitados pelo jogador, pois levam a situações estimuladoras do comportamento de risco para consumo de álcool. A publicação 
está na revista PloS One (LINDGREN K.P., et al, 2015). A segunda publicação, na revista JMIR Serious Games, é relativa à mesma temática e avalia outro jogo, no caso, o Shots Game, que objetiva modificar o nível de atenção cognitiva do jogador, desviando-a de situações estimuladoras de comportamento de risco para álcool (BOENDERMAKER, W. J., et al, 2016).

Observa-se que nessas duas publicações, não se considera formação de rede de coautoria, uma vez que somente Weirs, R. é autor comum a ambas as publicações.

Foi possível constatar que a temática dos “jogos sérios” tem sido foco de pesquisas na área da Saúde, evidenciando investigações sobre os atributos básicos de interação potencialmente relevantes para aprendizagem, como apontada por Bedwell et al (2012). Ainda em relação aos jogos sérios, nesta pesquisa foi evidenciado um periódico que se dedica exclusivamente ao tema, no caso, a JMIR Serious Games, descrita como sendo uma revista multidisciplinar sobre jogos e gamificação, incluindo simulação e realidade virtual imersiva para promoção da saúde, ensino, medicina, reabilitação e mudança social.

Para os oito autores identificados na figura 1, a força total dos vínculos de coautoria é maior entre os 4 autores na primeira rede, em vermelho, com força 6 da escala do VosViewer, seguido de 3 autores na rede verde, com força 4 e o autor Wiers, R. é representado com força nula de vínculo de co-autoria (cor azul).

Muito embora se perceba a importância dessa temática da Gamificação como metodologia de ensino nos cursos superiores, ainda não se evidencia nas publicações a existência de grandes redes colaborativas de pesquisas em inovação pedagógica no Ensino Superior, na área da Saúde.

Assim, podemos hipotetizar que estas evidências sobre o pequeno número de redes de co-autoria nas publicações identificadas na PubMed e a inexistência de redes colaborativas mais densas, com diversos co-autores comuns, reforçam a constatação de lacunas nas pesquisas, como apontado por Majuri; Koivisto; Hamari, (2018). Para os autores, há necessidade de se reformular os conteúdos instrucionais, bem como os elementos de jogos que poderiam dialogar na perspectiva de resultado desse movimento. Assim sendo, são necessários estudos com ênfase no design, aplicação prática e objetivos pretendidos com a atividade gamificada.

Em relação à dispersão das revistas que publicaram sobre gamificação no Ensino Superior (Figura 2) observou-se que o periódico que mais publicou foi o International 
Journal of Environmental Research and Public Health (vermelho) totalizando seis artigos, seguido dos periódicos JMIR Serious Games e do BMC Med Educ ambos com 4 publicações em cada um deles (amarelo).

Figura 2 - Dispersão de periódicos por frequência de publicação sobre Gamificação no Ensino Superior na área da Saúde

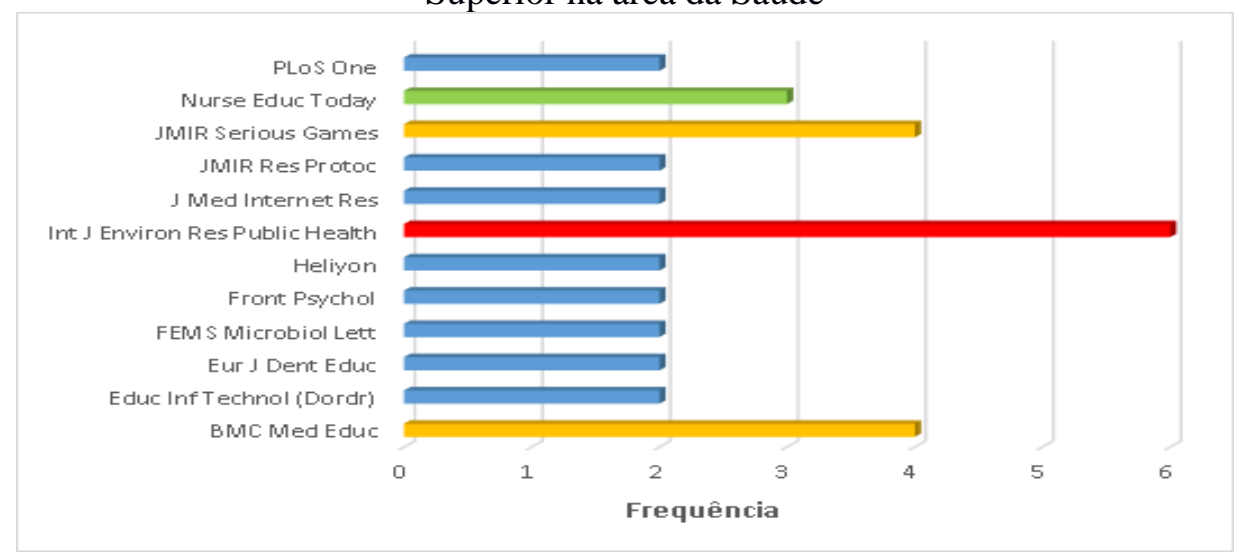

Fonte: Elaborado pelos autores

Com três publicações (verde) no Nurse Education Today e com apenas duas publicações (azul) nos periódicos Educ Inf Technol (Dordr); Eur J Dent Educ; FEMS Microbiol Lett; Front Psychol; Heliyon; J Med Internet Res; JMIR Res Protoc e na PLoS One (Figura 2).

A análise das conexões e relações no tema pesquisado está representada na figura 3. Pode-se identificar a frequência de ocorrências das palavras-chave citadas pelos autores como: gamificação, educação, jogos sérios, educação superior, motivação, e-learning, ensino, educação médica, e atividade física.

Observa-se na figura 3, que estudos mais recentes, do ano de 2020, têm forte ligação com as palavras-chave ensino e atividade física. Em 2019 percebe-se a concentração de pesquisas realizadas na temática de gamificação e educação com a preocupação com a motivação.

Figura 3 - Rede e clusters de palavras-chaves citadas nos artigos analisados 

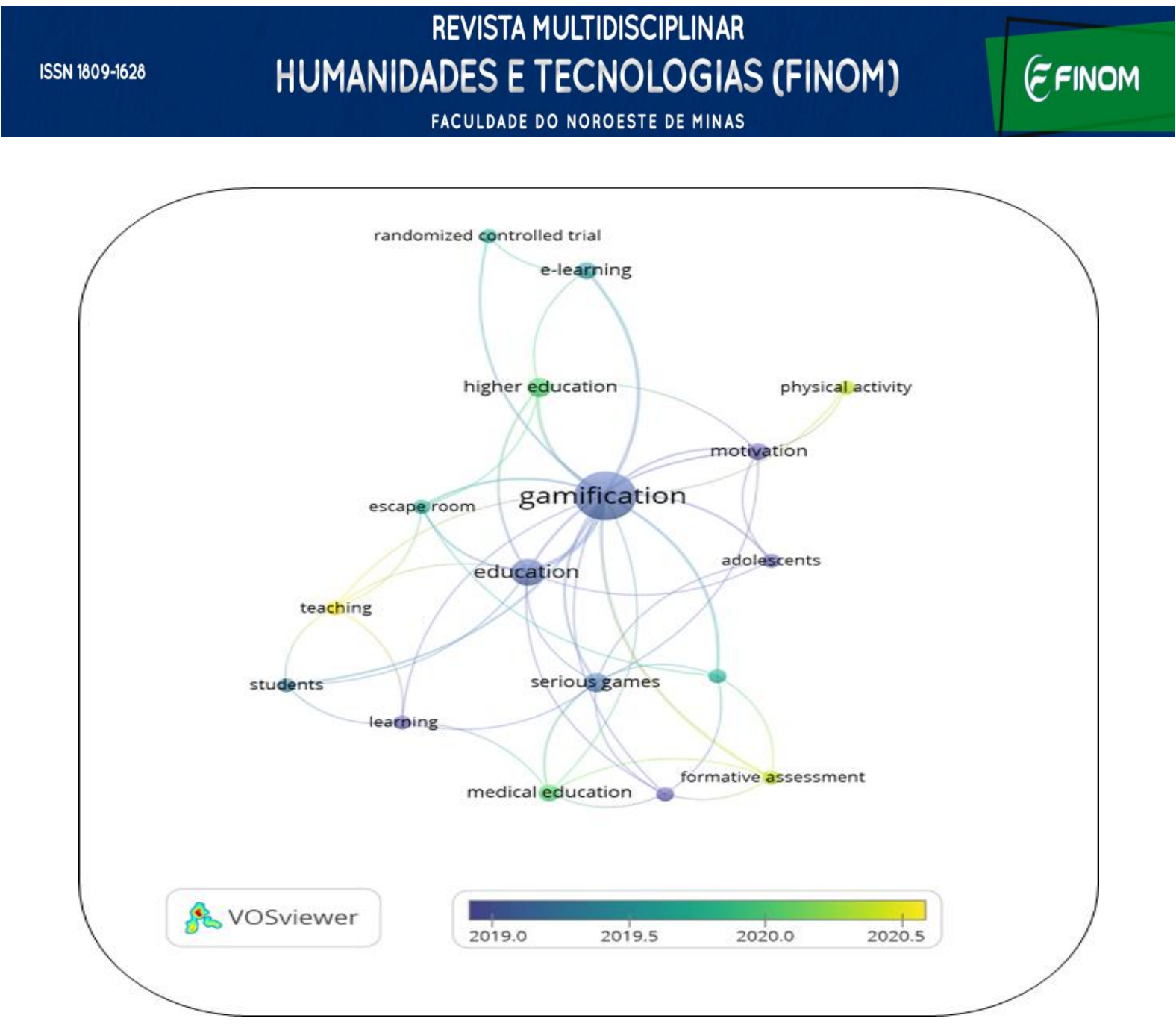

Fonte: elaborado pelos autores.

O primeiro uso documentado do termo gamificação data de 2008, porém até hoje persiste um debate sobre o significado exato do termo. Assim sendo, o significado aqui utilizado será aquele proposto por Deterding et al (2011, p. 2): “[...] gamificação é o uso de elementos característicos do universo dos jogos em qualquer outro contexto". A escolha por essa conceituação específica se deu pelo fato dela ser bem desenvolvida e amplamente aceita no meio acadêmico. Na definição em questão, entende-se como "outro contexto" situações como ensino acadêmico, treinamento de pessoas, marketing ou propaganda, por exemplo.

Em seu trabalho, Deterding et al (2011) também trata da definição de "jogo sério" (serious games), sendo igualmente seguido pelos demais autores. O “Jogo sério" envolve uma atividade que visa um propósito primário específico que não seja a interação lúdica e que possua em sua estrutura todos os elementos característicos de um jogo, como regras, objetivos, forma e apresentação. Em resumo, poderíamos dizer que a gamificação insere alguns elementos de jogo em uma determinada atividade e que os jogos sérios transformam a atividade em toda sua extensão em um jogo completo. 


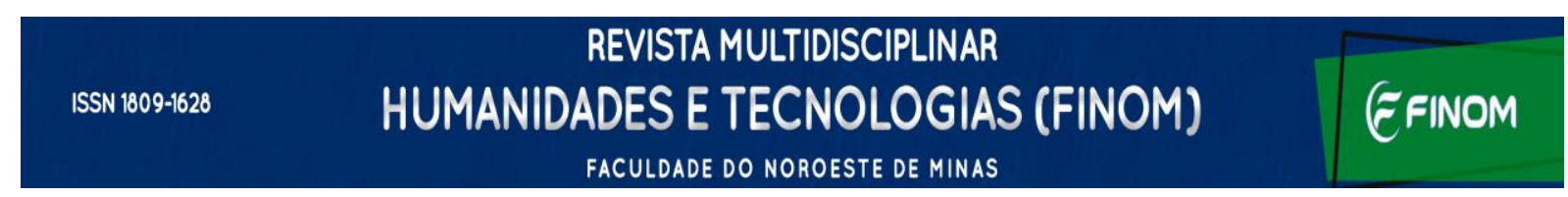

Como outra característica discriminatória, Landers (2014, p. 759) adiciona a definição já exposta o fato de que no contexto educacional os jogos sérios apresentam o conteúdo instrucional para o aluno dentro do jogo, enquanto na atividade gamificada o conteúdo instrucional foi introduzido em outra situação: "Embora os jogos possam também afetar a motivação ou o envolvimento do aluno, geralmente não é o propósito dos jogos sérios afetar essas características sem também fornecer ao aluno conteúdo instrucional". Apesar das diferenças citadas, esse autor afirma que o alto grau de sobreposição entre os dois temas torna impossível tratá-los como parte de constructos diferentes.

Em conjunto, os resultados desse estudo indicaram que a utilização da gamificação para ensinar conteúdos na área da saúde ainda é incipiente quando analisado o referido banco de dados. Além disso, observou-se que estes estudos se concentraram em três grandes áreas, nos levando a refletir até que ponto a formação de professores dessas áreas permitiu o uso dessa estratégia pedagógica. Entretanto, deve-se considerar futuramente outros bancos de dados para ampliar e verificar se os resultados são similares.

\section{Considerações finais}

Considerando o objetivo do estudo, qual seja, o de identificar a disseminação do conhecimento sobre os jogos digitais e a gamificação como inovação pedagógica no Ensino Superior na área da Saúde, evidenciamos que os estudos têm ligação com a área da Saúde principalmente na motivação, na educação médica e na área da atividade física.

Os principais periódicos que publicam sobre esta temática têm como escopo as Ciências Ambientais e a Engenharia, a Saúde Pública, na área multidisciplinar sobre jogos e gamificação, incluindo simulação e realidade virtual imersiva para educação na promoção da saúde. Foi possível identificar a relação entre o ensino e a educação médica na reabilitação e na mudança social. Não obstante, na área da Enfermagem, assim como nas Tecnologias Digitais de Informação e Comunicação. Para finalizar, neste tópico, nas Ciências Psicológicas, da Pesquisa Clínica às Ciências cognitivas, da percepção à consciência.

A despeito da importância da temática da gamificação como metodologia de ensino, ainda não há evidência de uma rede colaborativa de pesquisa em inovação pedagógica no Ensino Superior, na área da Saúde. 


\section{Referências}

ARAÚJO, C.A. Bibliometria: evolução histórica e questões atuais. Em Questão, v.12, n.1, p.11-32, 2006.

AZEVEDO, S. B.; PACHECO, V. A.; SANTOS, E. A. Dos. Metodologias ativas no ensino superior. Revista Docência do Ensino Superior, v. 9, n. 9, p. 1-22, Agosto, 2019.

ALMEIDA, Carla et al. Parental practices, preferences, skills and attitudes on food consumption of pre-school children: Results from Nutriscience Project. PloS one, v. 16, n. 5, p. e0251620, 2021.

AZEVEDO, José et al. A web-based gamification program to improve nutrition literacy in families of 3-to 5-year-old children: the Nutriscience Project. Journal of nutrition education and behavior, v. 51, n. 3, p. 326-334, 2019.

BAUMAN, Z. A cultura no mundo líquido moderno. Editora Schwarcz-Companhia das Letras, 2013.

BEDWELL, W. L. et al. Toward a Taxonomy Linking Game Attributes to Learning: An Empirical Study. Simulation \& Gaming, Orlando, v. 43, p. 729-760. 14 mai. 2012.

BOENDERMAKER, W. J. et al. Attentional bias modification with serious game elements: evaluating the Shots Game. JMIR serious games, v. 4, n. 2, p. e6464, 2016.

BUIJS-SPANJERS, K. R. et al. A web-based serious game on delirium as an educational intervention for medical students: randomized controlled trial. JMIR serious games, v. 6, n. 4, p. e17, 2018.

BUIJS-SPANJERS, K. R. et al. Reasons to Engage in and Learning Experiences From Different Play Strategies in a Web-Based Serious Game on Delirium for Medical Students: Mixed Methods Design. JMIR Serious Games, v. 8, n. 3, p. e18479, 2020.

CAILLOIS, R. Os jogos e os homens: a máscara e a vertigem. Lisboa: Cotovia, 2001.

DETERDING, Sebastian et al. Gamification: Toward a definition. In: CHI 2011 gamification workshop proceedings. Vancouver BC, Canada, 2011. p. 12-15.

DRUCKER, P.A. Nova sociedade das organizações. In: HOWARD, R. (Org.) Aprendizado organizacional. p. 1-7. Rio de Janeiro: Campus, 2000.

HUIZINGA, J. Homo ludens: o jogo como elemento da cultura. 4. ed. São Paulo: Editora Perspectiva, 2000.

LANDERS, R. N. Developing a Theory of Gamified Learning: Linking serious games and gamification of learning. Simulation \& Gaming, v. 45, n. 6, p. 752-768, 2014. 


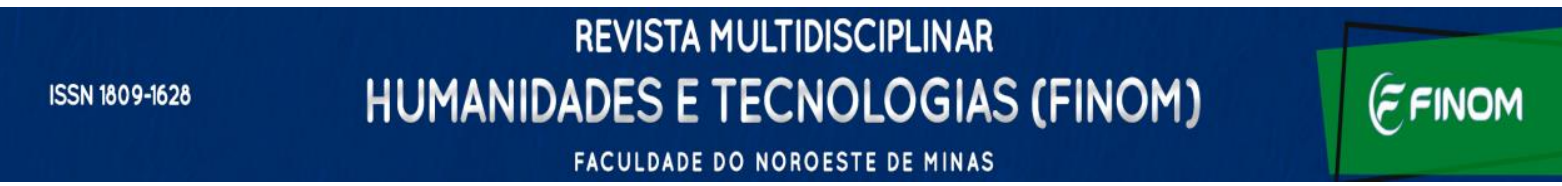

LINDGREN, K. P. et al. Attempted training of alcohol approach and drinking identity associations in US undergraduate drinkers: Null results from two studies. PloS one, v. 10, n. 8, p. e0134642, 2015.

LOBO DA COSTA, N. M.; RAMOS, M. A. S. Práticas Inovadoras com Tecnologias Digitais na Formação Inicial de Professores. Revista Sergipana de Matemática e Educação Matemática. ReviSeM, Ano 2020, N. 2, p. 1 - 18.

MAJURI, Jenni; KOIVISTO, Jonna; HAMARI, Juho. Gamification of education and learning: A review of empirical literature. In: Proceedings of the 2nd international GamiFIN conference, GamiFIN 2018. CEUR-WS, 2018.

OKUBO, Y. Bibliometric indicators and analyses of research systems methods and examples. Paris: OCDEGD, 1997.

ORTIZ-COLÓN, A. M.; JORDÁN, J.; AGREDAL, M. Gamification in education: an overview on the state of the art. Educação e Pesquisa, v. 44, n. 0, p. e173773, 2018.

SALEN, Katie; ZIMMERMAN, Eric. Regras do jogo: fundamentos do design de jogos (vol. 3). Editora Blucher, 2012.

SILVA, M.R..; HAYASHI, C.R.M..; HAYASHI, M.C.P.I. Análise bibliométrica e cientométrica: desafios para especialistas que atuam no campo. In CID Rev. Ciênc. Inform. Documentação, v.2, n.1, p.110-129, 2011.

WILSON, Katherine A. et al. Relationships between game attributes and learning outcomes: Review and research proposals. Simulation \& gaming, v. 40, n. 2, p. 217-266, 2009. 